\title{
An Iterative Discriminant Analysis Method to Search for the Higgs Particle at LEP2
}

\author{
T.G.M.Malmgren and K.E.Johansson
}

Fysikum, Stockholm University, Box 6730, S-113 85 Stockholm, Sweden

\begin{abstract}
The search for the Higgs boson in $\mathrm{e}^{+} \mathrm{e}^{-}$collisions at energies above the $\mathrm{Z}^{0}$ mass requires an efficient method to reject the many background channels. We have investigated an iterated discriminant analysis to detect the signal of a Higgs boson produced in association with neutrinos. An efficiency for a $70 \mathrm{GeV} / \mathrm{c}^{2} \mathrm{Higgs}$ particle of $50 \%$ and a background rejection factor of 290000 have been achieved. The optimization and performance of the iterated discriminant analysis are described.
\end{abstract}

To be submitted to Nuclear Instruments and Methods 



\section{Introduction}

Recently at LEP, the center of mass energy increased from $91 \mathrm{GeV}$ to $161 \mathrm{GeV}$ and then $172 \mathrm{GeV}$. At these energies, a larger signal over background ratio is expected for the production of a Higgs particle, but with a considerably larger number of background channels present. The Higgs particle is expected to be produced through the Bjorken process[1] where a virtual $\mathrm{Z}^{0}$ radiates the $\mathrm{H}^{0}$ and decays into a pair of fermions.

$$
\begin{array}{lll}
e^{+}+e^{-} \rightarrow Z^{0} \rightarrow \quad & Z^{0 *}+\quad H^{0} \\
& \longleftrightarrow \nu \bar{\nu} \quad \longrightarrow \mathrm{b} \overline{\mathrm{b}}
\end{array}
$$

We focus on the channel where the $\mathrm{H}^{0}$ decays into a pair of heavy quarks and the $\mathrm{Z}^{0}$ decays to a pair of neutrinos. The $\mathrm{H}^{0}$ predominantly decays into a $\mathrm{b}$ quark pair which is boosted due to the $\mathrm{H}^{0}$ movement. This gives the signature of isolated missing energy and acollinear jets of particles from the $b$ quarks. Methods for studying this process in $\mathrm{e}^{+} \mathrm{e}^{-}$ collisons at $\sqrt{s}=91 \mathrm{GeV}$ are described in $[2,3,4]$.

\section{Traditional selection and Discriminant Analysis}

In order to obtain a good separation between signal and background, a separating surface is searched for in the feature space. The latter is defined as the multi-dimensional space spanned by the event variables. Sequential cuts could be visualized as a rectangular box in this space. Linear discriminant analysis (DA)[5] may be visualized as a multi-dimensional hyperplane. However, the optimal separative surface is, in general, non-linear. Hence, we expand the linear DA to a non-linear by going from the first degree $\mathrm{D}_{1}=\mathrm{x}^{T} \mathbf{a}$ to the second degree $\mathrm{D}_{2}=\mathrm{x}^{T}(\mathbf{a}+\mathbf{B x})$, where the scalar $\mathrm{D}$ is the output, the transpose vector $\mathbf{x}^{T}$ is the input feature variables while the vector $\mathbf{a}$ and the matrix $\mathbf{B}$ are the coefficients to be determined in the optimization. By expanding $\mathbf{a}$ to contain the elements of $\mathbf{B}$ and by expanding $\mathrm{x}$ to contain the elements of $\mathrm{xx}^{T}$, the linear deduction of the coefficients may be used. The two criteria, that the local variance should be as small as possible while the separation should be as large as possible, could be expressed such that the ratio

$$
R=\frac{\left(\mathbf{a}^{T} \boldsymbol{\Delta} \mu\right)^{2}}{\mathbf{a}^{T} \mathbf{V a}}
$$

should be maximized where $\mathbf{V}$ is the variance matrix and $\boldsymbol{\Delta} \mu$ the mean differences between the signal and the background. This leads to $\mathbf{a}=\mathbf{V}^{-1} \boldsymbol{\Delta} \mu$.

When dealing with $\mathrm{N}_{s}$ signal and $\mathrm{N}_{b}$ background channels, the variance matrix used, $\mathbf{V}$, is set to be the sum of the individual variance matrices, $\mathbf{V}_{i}$, from each channel scaled with their cross section $\sigma$

$$
\mathbf{V}=\frac{1}{\sigma_{b, t o t}} \sum_{i=1}^{N_{b}} \sigma_{b, i} \mathbf{V}_{b, i}+\frac{1}{\sigma_{s, t o t}} \sum_{i=1}^{N_{s}} \sigma_{s, i} \mathbf{V}_{s, i}
$$

It is split into two separate sums in order to give the same importance to signal and background. 


\section{Analysis}

\subsection{Event samples and preselection}

The data were collected with the DELPHI detector[6] during 1996. A total integrated luminosity of about 10 events per pb was collected at $\sqrt{s}=161 \mathrm{GeV}$ and $\sqrt{s}=172 \mathrm{GeV}$, respectively. The optimization of the analysis method was based on simulated data for the Higgs signal and the many background channels. Background events were generated with PYTHIA[11] except for two photon interactions which were generated with TWOGAM[7]. Signal events were generated by HZHA[8]. All events were then run through the DELPHI Monte Carlo simulation routines $[9,10,11,12]$.

At energies above the $Z^{0}$ threshold, a multitude of background channels is expected. These processes can be seen in figure 1 . The cross-sections, the number of simulated events
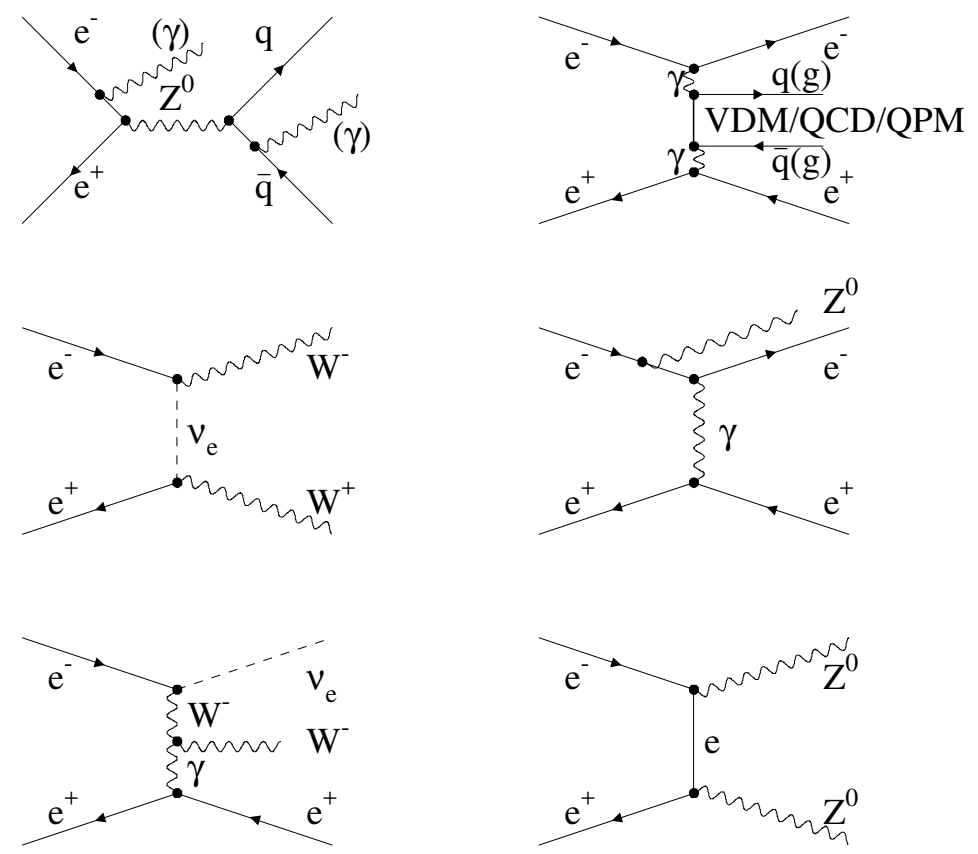

Figure 1: The main background channels.

and the corresponding luminosities for the background channels are listed in table 1 for the two collision energies. The samples marked with "bias" were simulated with special selections to increase the luminosity in those channels. The signal samples were generated at an $\mathrm{H}^{0}$ mass ranging from 45 to $70 \mathrm{GeV} / \mathrm{c}^{2}$ at $\sqrt{s}=161 \mathrm{GeV}$ and from 45 to $80 \mathrm{GeV} / \mathrm{c}^{2}$ 
Table 1: Simulated background channels and signal with cross sections $(\sigma)$, number of events and the corresponding luminosity

\begin{tabular}{|c|c|c|c|c|c|c|}
\hline \multirow{2}{*}{$\begin{array}{l}\text { Background- } \\
\text { process }\end{array}$} & \multicolumn{3}{|c|}{$\sqrt{s}=161 \mathrm{GeV}$} & \multicolumn{3}{|c|}{$\sqrt{s}=172 \mathrm{GeV}$} \\
\hline & $\sigma(\mathrm{pb})$ & $\mathrm{N}^{\circ} \mathrm{ev}$. & $\overline{\mathcal{L}(\epsilon v \cdot / p b)}$ & $\sigma(\mathrm{pb})$ & $\mathrm{N}^{\circ} \mathrm{ev}$. & $\overline{\mathcal{L}(e v \cdot / p b)}$ \\
\hline $\mathrm{q} \overline{\mathrm{q}}$ & 147 & 64033 & 436 & 121 & 59853 & 495 \\
\hline bias $(b \bar{b})$ & & & & 23.25 & 35833 & 1541 \\
\hline total & & & 436 & & & 2036 \\
\hline $\mathrm{We} \nu_{e}$ & 0.4 & 557 & 1393 & 0.48 & 713 & 1485 \\
\hline$W^{+} W^{-}$ & 3.4 & 4806 & 1414 & 12.3 & 14595 & 1187 \\
\hline$Z^{0} Z^{0}$ & 0.41 & 1343 & 3276 & 1.15 & 1929 & 1677 \\
\hline $\mathrm{Z}^{0} \mathrm{e}^{+} \mathrm{e}^{-}$ & 6.3 & 5960 & 946 & 98.2 & 75557 & 769 \\
\hline$\gamma \gamma(\mathrm{QPM})$ & 960 & 20279 & 21.1 & 1081 & 47296 & 43.8 \\
\hline bias & 148 & 12218 & 82.6 & & & \\
\hline total & & & 104 & & & 43.8 \\
\hline$\gamma \gamma(\mathrm{QCD})$ & 1880 & 19712 & 10.5 & 2048 & 34131 & 16.7 \\
\hline bias & 320 & 25671 & 80.2 & 351 & 13247 & 37.7 \\
\hline total & & & 90.7 & & & 54.5 \\
\hline$\gamma \gamma(\mathrm{VDM})$ & 6700 & 45247 & 6.75 & 7600 & 96302 & 12.7 \\
\hline bias & 521 & 37426 & 71.8 & 540 & 39542 & 73.2 \\
\hline total & & & 78.6 & & & 85.9 \\
\hline$\overline{\mathrm{H}^{0} \nu \bar{\nu} 65 \mathrm{GeV} / \mathrm{c}^{2}}$ & 0.659 & 1900 & 2883 & $\overline{0.944}$ & 1500 & 1589 \\
\hline $\mathrm{H}^{0} \nu \bar{\nu} 70 \mathrm{GeV} / \mathrm{c}^{2}$ & 0.165 & 2000 & 12121 & 0.735 & 2998 & 4079 \\
\hline
\end{tabular}

at $\sqrt{s}=172 \mathrm{GeV}$ in steps of $5 \mathrm{GeV} / \mathrm{c}^{2}$ with 1500 to 3500 events at each mass. The cross sections and luminosities for two mass points of the simulated signal with a 65 and a $70 \mathrm{GeV} / \mathrm{c}^{2}$ Higgs particle mass are shown in table 1 . Here we focus on the analysis at $\sqrt{s}=172 \mathrm{GeV}$.

In order to suppress rather trivial background channels not simulated, such as beamgas interactions, noise or cosmic particles and to remove a large part of the $\gamma \gamma$ processes where a relatively small part of the energy of the colliding electron positron pair goes into particle production, three cuts were made as a preselection before the variables were fed into the discriminant analysis. Events were kept if the number of tracks from charged particles was nine or more, if the energy from them was more than $10 \%$ of the center of mass energy and if the number of tracks from charged particles originating from the primary vertex, within $200 \mu \mathrm{m}$ in the plane transverse to the beam axis, was one or more. The preselection retained $91 \%$ of the $\mathrm{H}^{0}$ events for an $\mathrm{H}^{0}$ mass of $70 \mathrm{GeV} / \mathrm{c}^{2}$ while reducing the background with a factor 71 from 124000 to 1757 expected events.

\subsection{Variables}

Ten variables were selected

- the scaled acoplanarity, $\alpha$, (i.e. acoplanarity multiplied by $\min \left(\sin \left(\theta_{i}\right)\right)$ when the event is divided into two hemispheres by the thrust axis, where $\theta_{i}$ are the polar angles of the total momentum of respective hemisphere,

- the recoil mass, 
- the b-tag value estimating the b quark probability[14],

- the cosine of the polar angle of the (missing) momentum,

- the energy of charged particles,

- the energy of a hypothetical photon assumed to be lost in the beam-pipe,

- the ratio of the total energy within $30^{\circ}$ of the beam-axis to the total visible energy,

- the visible mass,

- the energy within $5^{\circ}$ and $20^{\circ}$ around the most isolated track,

- the momenta of the most isolated track in $\mathrm{x}$-direction.

The variables were then transformed to lie in $[0,1]$ through

$$
x \mapsto x^{\prime}=\frac{1}{2 \sigma_{b, t o t}} \sum_{i=1}^{N_{b}} \frac{\sigma_{b, i}}{n_{b, i}} \sum_{j=1}^{n_{b, i}} \theta\left(x-x_{b, i j}\right)+\frac{1}{2 \sigma_{s, t o t}} \sum_{i=1}^{N_{s}} \frac{\sigma_{s, i}}{n_{s, i}} \sum_{j=1}^{n_{s, i}} \theta\left(x-x_{s, i j}\right)
$$

where $\theta$ is Heaviside's step-function. This is done to reduce wide distributions with long tails. Also, it transforms variables with narrow peaks to be distributed along the whole interval. It is split into two separated sums in order to let the signal have the same importance as the background. An example of this transformation can be seen in figure 2, which shows the real data, the simulated data of the background and the Higgs particle signal. The latter two were normalized to the integrated luminosity of real data. Figure $2 \mathrm{a}$ shows the original distribution, figure $2 \mathrm{~b}$ the transformed distribution while figure $2 \mathrm{c}$ shows the transformation function. The signal sample consisted of two Higgs particle samples of mass 65 and $70 \mathrm{GeV} / \mathrm{c}^{2}$ respectively. The masses were chosen to lie in the interesting search region according to the earlier results of the four LEP experiments[15]. The background sample consisted of the 12 background channels listed in table 1.

\subsection{Iterated discriminant analysis in the $\mathbf{H}^{0}$ search}

At collision energies above the $Z^{0}$ peak, several background channels with rather different properties have to be considered. Hence, it is difficult to reduce sufficiently all background channels with one DA. With this multitude of background processes with very different cross sections, we explore their suppressions with an Iterated Discriminant Analysis (IDA) of second degree. The first iteration will be dominated by the largest background channels. The next iterations will deal with a much smaller, but potentially more harmful, number of background events. In each iteration, the cut in the discriminant output is made so that a predefined fraction of the signal is kept. The IDA consists of the following four steps:

1 calculate the variance matrix $\mathbf{V}$ and mean differences $\boldsymbol{\Delta} \mu$ of the remaining events at this stage,

2 calculate $\mathbf{a}=\mathbf{V}^{-1} \boldsymbol{\Delta} \mu$ 

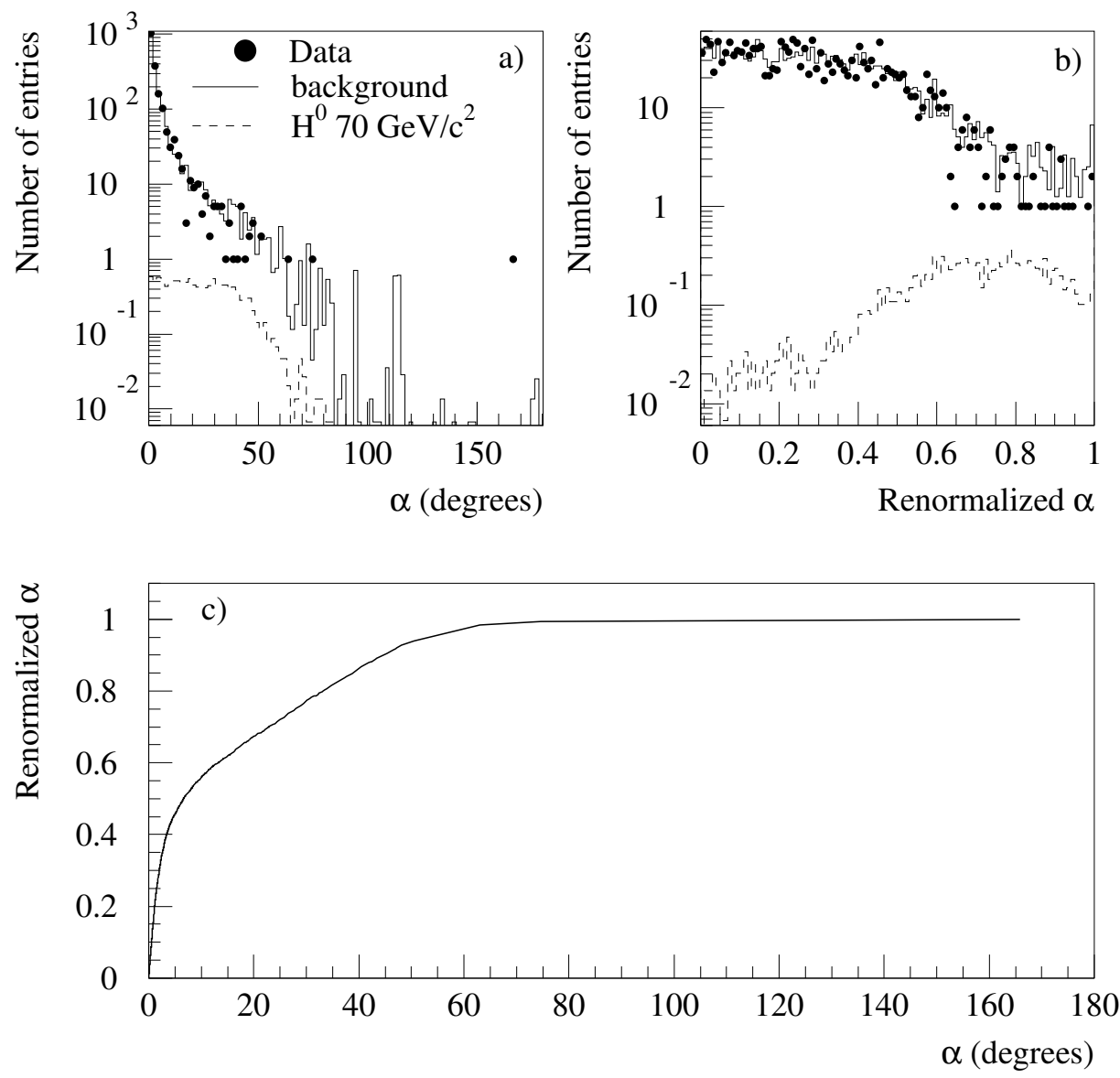

Figure 2: The scaled acoplanarity before and after transformation. In the lower plot, the transformation function is shown. 
3 cut data in the DA output $\mathrm{D}_{2}$ at the desired $\mathrm{H}^{0}$ step efficiency $\left(\epsilon_{s}\right)$

4 if the total expected background is larger than the desired number of events, then iterate from step 1

The performance depends on the number of variables used, the step efficiency chosen for each iteration, the number of iterations and the tolerated amount of background. The two Higgs samples listed in table 1 were used for all optimizations.

To study the optimal number of variables to use, six different analyses were made with the number of variables ranging from two to ten. The $\mathrm{H}^{0}$ efficiency as a function of the background rejection factor was used as a measurement of the performance. In figure 3, the results from this study can be seen. The fitted curves in the figure are merely guides

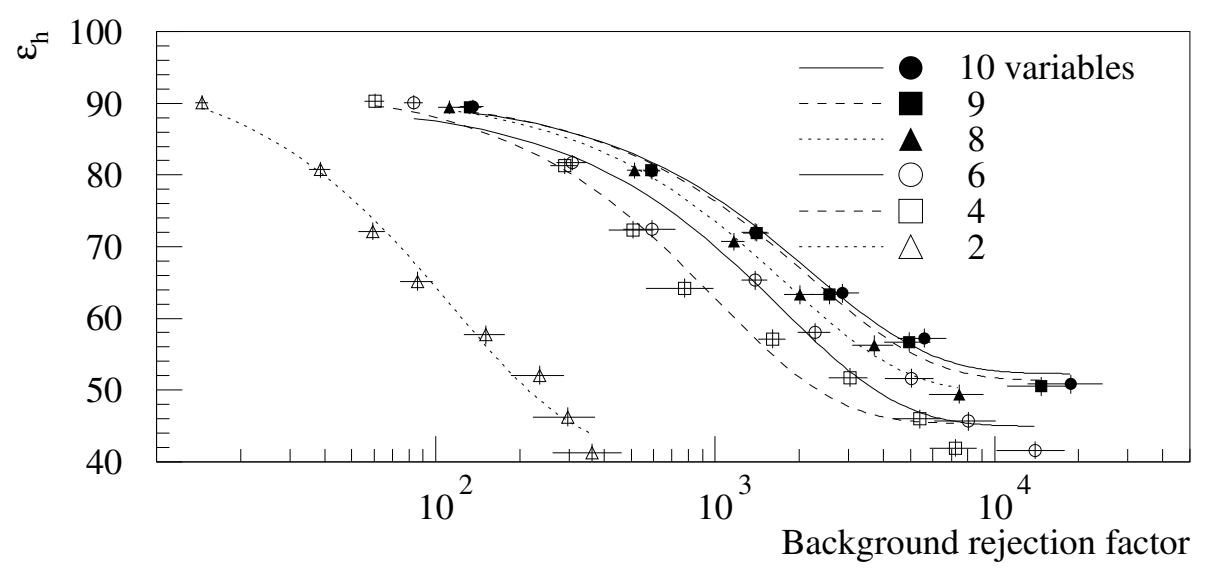

Figure 3: Efficiency for a $70 \mathrm{GeV} / \mathrm{c}^{2}$ Higgs particle as a function of the background rejection factor for a different number of variables and the eight iterations.

for the eye and connect the results obtained for the consecutive eight iterations. The performance improves with a larger number of variables. For nine and ten variables, the performance is very similar for background rejection factors up to around $2 \times 10^{3}$, while somewhat better for ten variables and larger background rejection factors. For the subsequent performance tests, we use ten variables.

To determine the second parameter, $\epsilon_{s}$, several analyses were made with various values of $\epsilon_{s}$. The background rejection factor versus the $\mathrm{H}^{0}$ efficiency was used to measure the performance of the IDA. The results are shown in figure 4 for eight iterations with $\epsilon_{s}=95 \%$, six iterations with $\epsilon_{s}=90 \%$ and four iterations with $\epsilon_{s}=85 \%$. After a few iterations the performance is approximately the same for any $\epsilon_{s}$. However, for large $\epsilon_{s}$, greater than around $95 \%$, a large number of steps is required to reach a satisfying background level. To select the optimal $\epsilon_{s}$, the number of steps required also has to be considered. Five iterations with $\epsilon_{s}=90 \%$ and three iterations with $\epsilon_{s}=85 \%$ give very similar performance. For the subsequent tests we use both $85 \%$ and $90 \%$ step efficiency. 


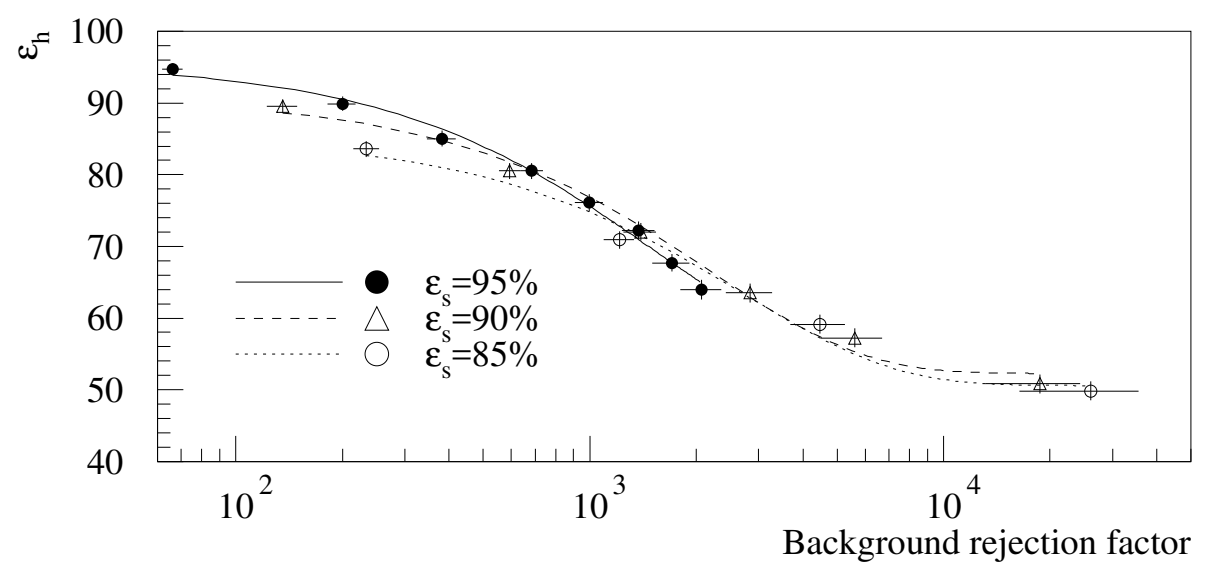

Figure 4: Efficiency for a $70 \mathrm{GeV} / \mathrm{c}^{2}$ Higgs particle as a function of the background rejection factor for various values of $\epsilon_{s}$.

\section{Uncertainties}

The three main uncertainties are due to 1) an optimization overfit of the samples, 2) not having enough events to generalize the sample or 3 ) due to differences between real and simulated data. The first two can be dealt with by using an independent test sample. To investigate these effects, several analyses were done with different fractions of data used for optimization. The amount of background used varied from $10 \%$ to $100 \%$ for the background channels $q \overline{\mathrm{q}}$, We $\nu_{e}, \mathrm{~W}^{+} \mathrm{W}^{-}$and $\mathrm{Z}^{0} \mathrm{Z}^{0}$. The test sample size then varied from $90 \%$ to $0 \%$ (the complement of the optimization sample.) In figure 5 the total number of expected background events can be seen as a function of the percentage of data used for three iterations and $\epsilon_{s}=85 \%$ based on the optimization and test samples. The signal efficiencies as well as the individual expected number of events from the three most potential background channels, $q \bar{q}, W e \nu_{e}$ and $\mathrm{W}^{+} \mathrm{W}^{-}$, are shown.

The effect of overfitting is apparent when the expected background increases from the optimization sample to the test sample. The effect of not generalizing well enough is seen by the slope of the expected background. With very small optimization samples, there is a lack in generalization of the samples. For larger optimization samples, the generalization improves and the real performance, the one given by the test sample, approaches the one given by the optimization sample. In figure 6 the same plot can be seen for five steps with $90 \%$ step efficiency. The performance of the analysis with five steps and $\epsilon_{s}=90 \%$ is very similar to the one with three steps and $\epsilon_{s}=85 \%$. The background estimates also show very similar behaviour. However, there is a tendency for the analysis with fewer steps, and consequently fewer coefficients to determine, to reach a better optimization and have a smaller effect from overfitting. 


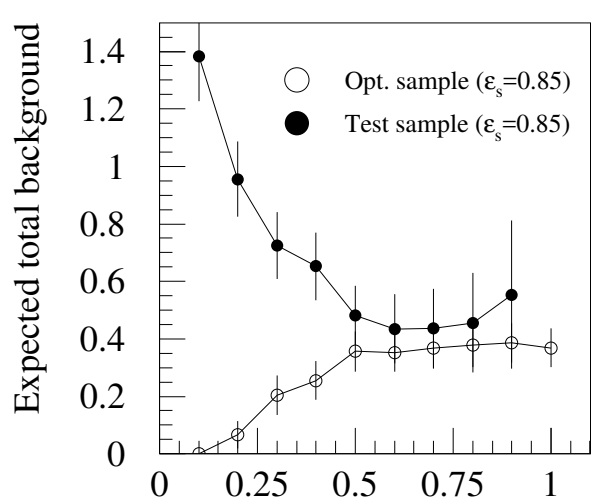

Fraction of total background used

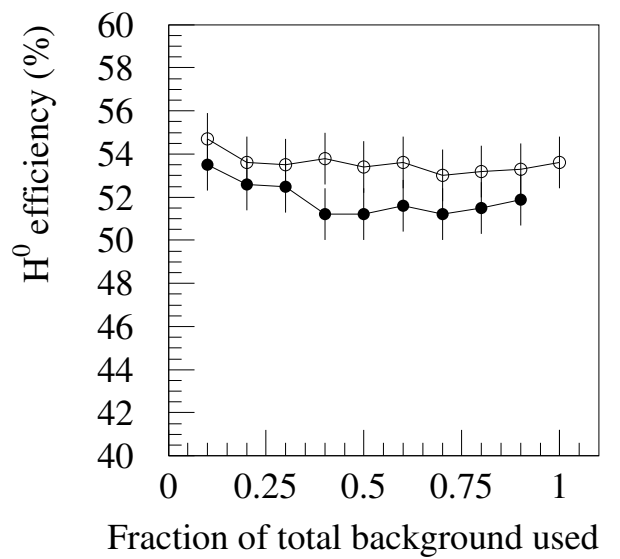

Fraction of total background used
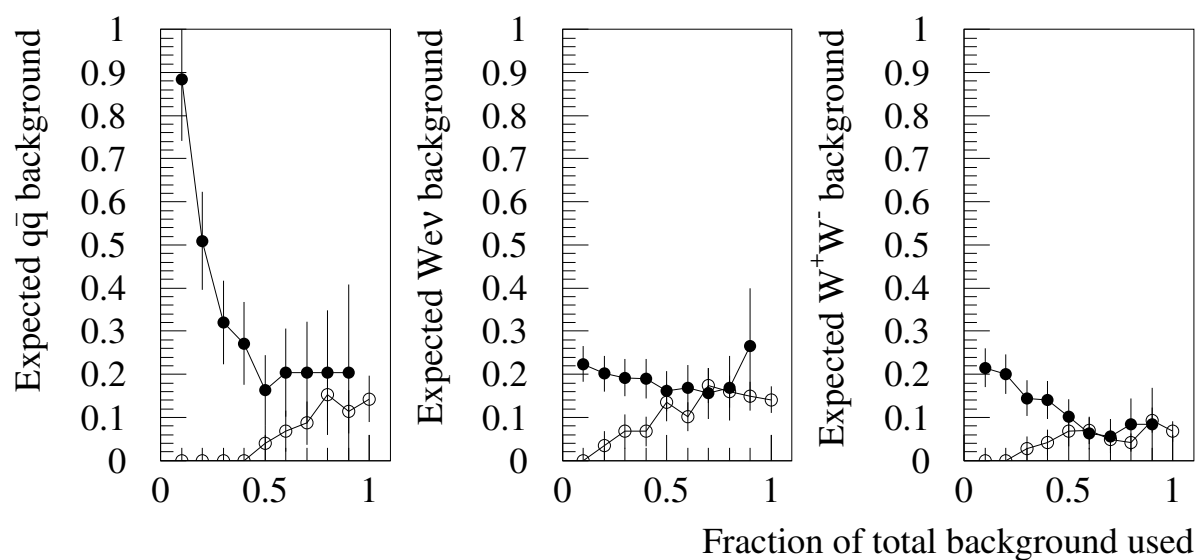

Figure 5: Expected events after the analyses as a function of the fraction of the total background used for optimizing. The test samples consisted of the remaining number of events. Three iterations with $\epsilon_{s}=85 \%$ were performed. 


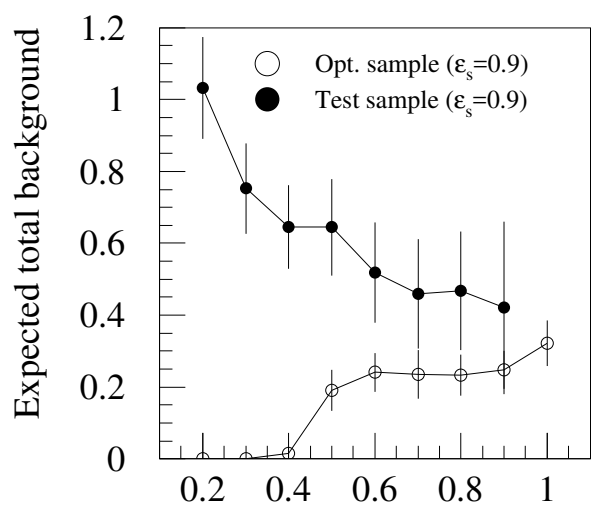

Fraction of total background used

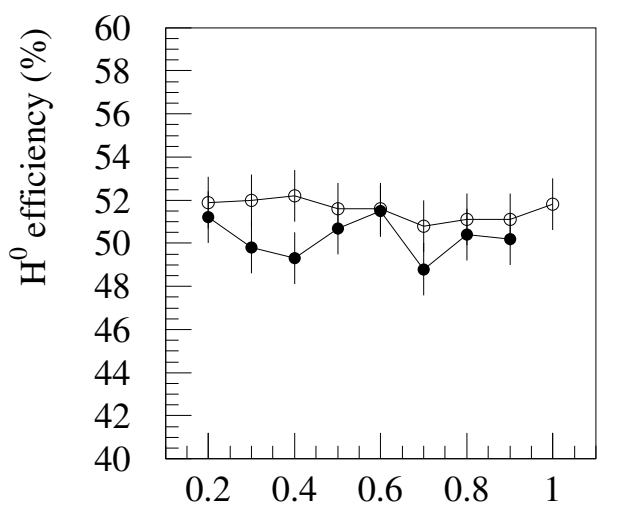

Fraction of total background used
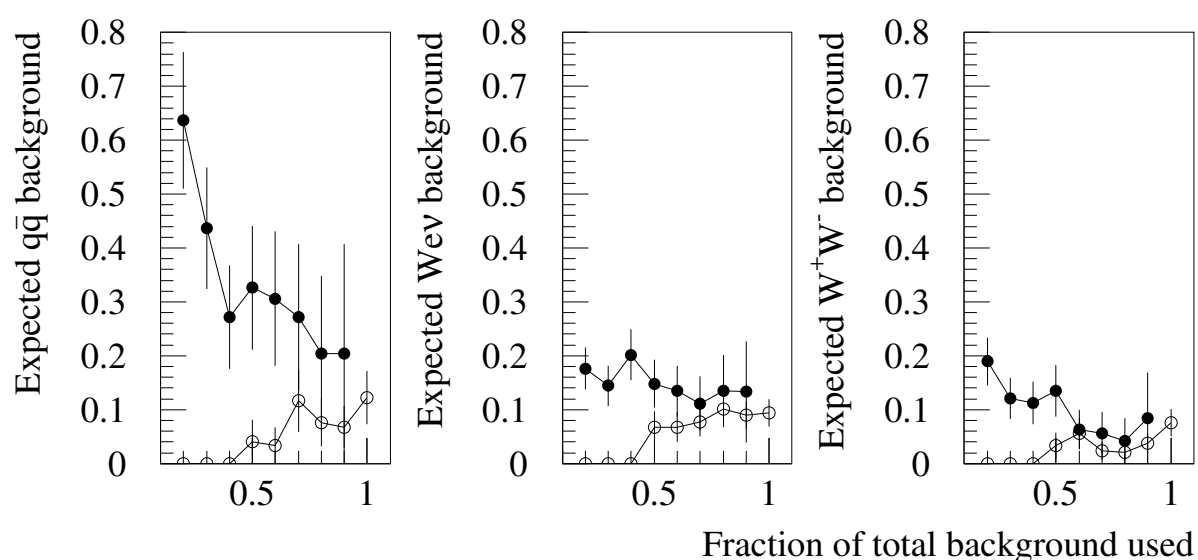

Figure 6: Expected events after the analyses as a function of the fraction of the total background used for optimizing. The test samples consisted of the remaining number of events. Five iterations with $\epsilon_{s}=90 \%$ were performed. 


\subsection{Data and simulation comparisons}

The optimization of the analysis is based on simulated events. An imperfect event simulation will lead to uncertainties in the estimate of efficiencies and background levels. We therefore evaluate what the effect of varying the simulated distributions have on Higgs particle detection efficiencies and background levels. The differences between real and simulated data were dealt with by looking at the mean values, and the width of the distributions in the ten variables for real and simulated data after the preselection. The simulated data were shifted randomly in each variable between the mean values as well as between the widths. These changes were made 2000 times and the analysis was redone using the same coefficients achieved using the original simulated data.

Figure 7 shows the distribution of the $70 \mathrm{GeV} / \mathrm{c}^{2}$ Higgs efficiencies obtained for the 2000 different changes. The width of the distribution is $0.85 \%$. However, the estimated

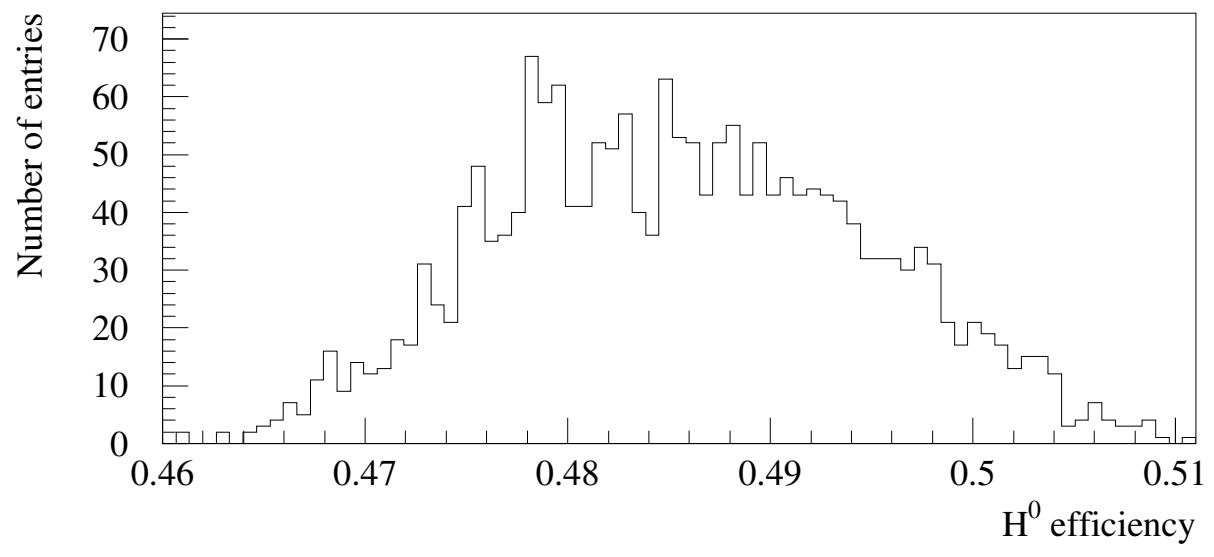

Figure 7: Selection efficiency for a $70 \mathrm{GeV} / \mathrm{c}^{2}$ Higgs particle for 2000 different changes at $\mathrm{E}_{c m s}=172 \mathrm{GeV}$.

efficiency also shows a shift from the original value $50.3 \%$. For a conservative estimate of the uncertainty we use both the width of the distribution and the shift by adding them. This leads to an uncertainty estimate of ${ }_{-2.6}^{+0} \%$. The width of the distributions ranges from 0.5 to $1.3 \%$ at $172 \mathrm{GeV}$ and from 1.5 to $2.8 \%$ at $161 \mathrm{GeV}$ while the shift ranges from -1.7 to $+1.5 \%$ at $172 \mathrm{GeV}$ and from -4.9 to $+2.5 \%$ at $161 \mathrm{GeV}$. The real and simulated data agree rather well for the $172 \mathrm{GeV}$ run, while there are some discrepancies for the $161 \mathrm{GeV}$ run. This fact is reflected in the larger uncertainties estimated for the latter.

\section{$5 \quad$ Final results}

In $[16,17]$, ten variables were selected, $\epsilon_{s}$ was chosen to $90 \%$ and five steps were done. The two Higgs samples listed in table 1 were used for optimization. All signal and background data were run through the analysis using $75 \%$ of the samples for optimization and $25 \%$ for testing. Table 2 shows the test sample results obtained with this method while the effect on each background channel can be seen in table 3 . 
Table 2: Effect of the IDA selections at $\mathrm{E}_{c m s}=172 \mathrm{GeV}$. Efficiencies are given for a $70 \mathrm{GeV} / \mathrm{c}^{2}$ Higgs particle.

\begin{tabular}{|l|r|c|c|}
\hline Selection & Data & Background & Efficiency (\%) \\
\hline pre & 1898 & $1757 \pm 16$ & 90.7 \\
step 1 & 9 & $8.5 \pm 0.72$ & 78.9 \\
step 2 & 1 & $2.4 \pm 0.35$ & 72.1 \\
step 3 & 0 & $0.97 \pm 0.23$ & 64.6 \\
step 4 & 0 & $0.51 \pm 0.17$ & 55.9 \\
step 5 & 0 & $0.38 \pm 0.14_{-0.08}^{+0.01}$ & 50.3 \\
\hline
\end{tabular}

Table 3: Effect of the IDA selections at $\mathrm{E}_{c m s}=172 \mathrm{GeV}$ for the different background channels.

\begin{tabular}{|l|c|c|c|c|c|c|}
\hline selection & $\mathrm{q} \overline{\mathrm{q}}$ & $\mathrm{We} \nu_{e}$ & $\mathrm{~W}^{+} \mathrm{W}^{-}$ & $\mathrm{Z}^{0} \mathrm{Z}^{0}$ & $\mathrm{Z}^{0} \mathrm{e}^{+} \mathrm{e}^{-}$ & $\gamma \gamma$ \\
\hline pre & 1149 & 3.2 & 109 & 8.5 & 22 & 457 \\
step 1 & 5.0 & 1.2 & 2.1 & 0.17 & 0 & 3.4 \\
step 2 & 1.1 & 0.54 & 0.71 & 0.024 & 0 & 0 \\
step 3 & 0.49 & 0.32 & 0.13 & 0.024 & 0 & 0 \\
step 4 & 0.26 & 0.19 & 0.067 & 0 & 0 & 0 \\
step 5 & 0.21 & 0.11 & 0.067 & 0 & 0 & 0 \\
\hline
\end{tabular}

The signal efficiencies for different assumed $\mathrm{H}^{0}$ masses are listed in table 4 and shown in figure 8. A high efficiency is achieved over the important mass range.

Table 4: Efficiency of the Higgs boson selection as a function of the particle mass. The first uncertainty is statistical, the second is the worst case estimate from data and simulation differences.

\begin{tabular}{|c|c|c|}
\hline $\mathrm{m}_{h}\left(\mathrm{GeV} / \mathrm{c}^{2}\right)$ & $\begin{array}{c}\text { Efficiency }(\%) \\
\sqrt{s}=161 \mathrm{GeV}\end{array}$ & $\begin{array}{c}\text { Efficiency }(\%) \\
\sqrt{s}=172 \mathrm{GeV}\end{array}$ \\
\hline 45 & $23.0 \pm 0.9_{-7.4}^{+0}$ & $39.0 \pm 0.8_{-0}^{+2.7}$ \\
50 & $35.5 \pm 1.1_{-5.9}^{+0}$ & $46.9 \pm 1.3_{-0}^{+2.3}$ \\
55 & $47.8 \pm 1.1_{-3.3}^{+0.3}$ & $54.1 \pm 1.3_{-1.1}^{+1.0}$ \\
60 & $57.8 \pm 1_{-2.7}^{+0.2}$ & $60.0 \pm 1_{-1.1}^{+0}$ \\
65 & $57.6 \pm 1.5_{-2.5}^{+2.4}$ & $58.9 \pm 1.7_{-1.4}^{+0}$ \\
70 & $49.3 \pm 1.4_{-0}^{+4.4}$ & $50.3 \pm 1.2_{-2.6}^{+0}$ \\
75 & & $35.8 \pm 0.9_{-2.7}^{+0}$ \\
80 & & $17.1 \pm 0.7_{-1.9}^{+0}$ \\
\hline
\end{tabular}

The same procedure, variables, step efficiency and number of steps were used to optimize and retrieve the coefficients for the $\sqrt{s}=161 \mathrm{GeV}$ data. The amount of real and simulated data as well as the efficiency for a $60 \mathrm{GeV} / \mathrm{c}^{2}$ Higgs particle can be seen in table 5. The Higgs particle efficiencies for different masses are listed in table 4.

One candidate was found in the $\sqrt{s}=161 \mathrm{GeV}$ data and is described in detail in [18]. 


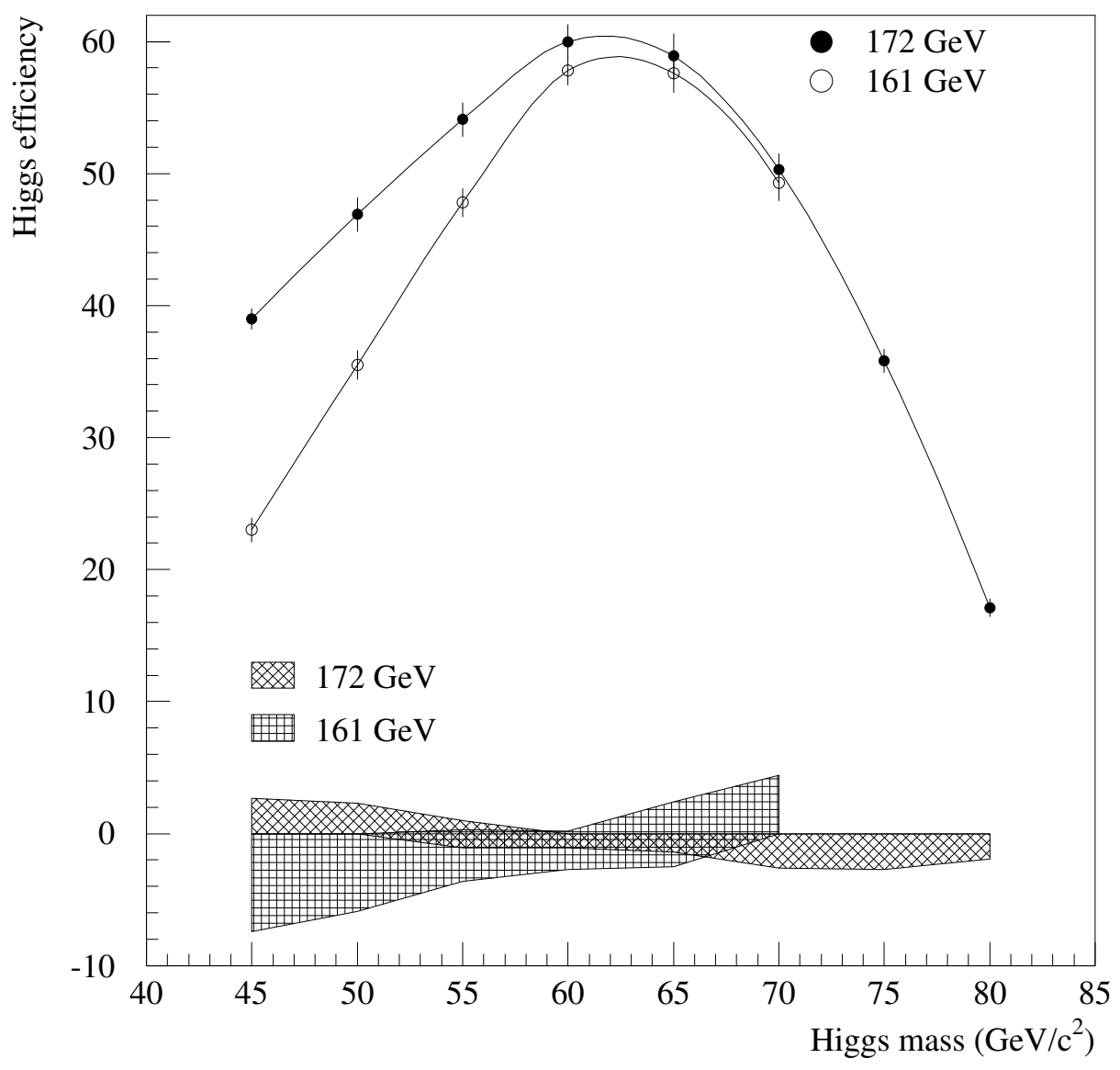

Figure 8: Higgs efficiencies as a function of the Higgs mass. The error bars are the statistical errors, while the hatched areas corresponds to the estimate of the uncertainty due to the differences between real and simulated data.

Table 5: Effect of the IDA selections at $\mathrm{E}_{c m s}=161 \mathrm{GeV}$. Efficiencies are given for a $60 \mathrm{GeV} / \mathrm{c}^{2}$ Higgs particle.

\begin{tabular}{|l|r|c|c|}
\hline Selection & Data & Background & Efficiency (\%) \\
\hline pre & 2181 & $1886 . \pm 21$. & 90.1 \\
step 1 & 18 & $7.5 \pm 0.76$ & 83.7 \\
step 2 & 4 & $2.3 \pm 0.42$ & 76.6 \\
step 3 & 1 & $1.4 \pm 0.33$ & 69.2 \\
step 4 & 1 & $1.0 \pm 0.29$ & 62.4 \\
step 5 & 1 & $0.47 \pm 0.19_{-0.01}^{+0.49}$ & 57.8 \\
\hline
\end{tabular}




\section{The program}

A computer program doing the analyses was developed to automatize the procedure. As input, it requires two or three data statements to define the cross sections and the effect of the preselection. The program also requires three parameters which are the number of steps, efficiency for each step and percentage of the simulated background samples to use. The stopping criterion for the iteration process is either that the desired background level has been reached or, if the previous cannot be reached, the predefined number of steps has been done. The output is a list of the different signal and background channels with the number of events remaining and their correspondence in the real data sample as well as the total background expected in the real data sample and the signal efficiency. A typical run with 113000 events, five steps and ten variables requires $40 \mathrm{~s}$ on a DEC 3000-M900. A more detailed description of the program can be found in [19].

\section{Conclusions}

Assuming a Higgs particle mass of $65 \mathrm{GeV} / \mathrm{c}^{2}$, three events were expected by the Standard Model to be produced in 1996 in the neutrino channel in the DELPHI detector at LEP at $100 \%$ signal efficiency. A total integrated luminosity of ten events per pb was collected at center of mass energies 161 and $172 \mathrm{GeV}$. A preselection is applied to remove the bulk of irrelevant- and two photon interaction background channels. An Iterated non-linear Discriminant Analysis (IDA) is applied to remove the Higgs particle like background.

A total background of $0.47 \pm 0.19$ (stat.) ${ }_{-0.01}^{+0.49}$ events is expected (a rejection factor of $210000)$ at $\sqrt{s}=161 \mathrm{GeV}$ and a Higgs particle efficiency of $57.8 \%$ is achieved for a Higgs particle mass of $60 \mathrm{GeV} / \mathrm{c}^{2}$. At $\sqrt{s}=172 \mathrm{GeV}$, a total background of $0.38 \pm 0.14$ (stat. $)_{-0.08}^{+0.01}$ is expected (a rejection factor of 290000 ) while a Higgs particle efficiency of $50.3 \%$ for a Higgs particle mass of $70 \mathrm{GeV} / \mathrm{c}^{2}$ is achieved. The second uncertainty is the estimate from data and simulation differences. At $\sqrt{s}=161 \mathrm{GeV}$, the differences between data and simulation are larger, which is reflected in the uncertainty estimate. One candidate was found in the 1996 data. It is explained more in detail in [18].

The IDA proves to be a very effective tool in the Standard Model Higgs boson search in the neutrino channel.

\section{Acknowledgments}

We are greatly indebted to our DELPHI collaborators and to the funding agencies for their support in building and operating the DELPHI detector. Many thanks to Dr.R.Keränen who processed real and simulated data and optimized the variables used. Thanks to Dr.K.Hultqvist and Dr.Ch.Walck for valuable discussions.

\section{References}

[1] P. J. Franzini et al., Z Physics at LEP, CERN 89-08 Vol.2.

[2] K. Hultqvist, R. Jacobsson, K. E. Johansson, Using a neural network in the search for the Higgs boson, Nucl. Instr. and Meth. 364 (1995) 193. 
[3] T. G. M. Malmgren and K. E. Johansson, Use of Discriminant Analysis in Search of a neutral Higgs boson, DELPHI 97-59 PHYS 709, Submitted to Nucl. Instr. and Meth.

[4] DELPHI Collaboration, Search for the Standard Model Higgs Boson in $Z^{0}$ Decays, Nucl. Phys. B421 (1994) 3.

[5] M. G. Kendall and A. Stuart, The Advanced Theory of Statistics, Volume 3.

[6] DELPHI Collaboration, The DELPHI detector at LEP, Nucl. Instr. and Meth. A303 (1991) 233.

[7] S. Nova, A. Olchevski and T. Todorov, MONTE-CARLO Event Generator for two Photon Physics, DELPHI 90-35 PROG 152.

[8] P. Janot, Event Generators for Discovery Physics - HZHA, Proceedings of "Physics at LEP2", Ed. G. Altarelli, T. Sjöstrand and F. Zwirner; CERN yellow report, CERN 96-01, Vol. 2 (1996) 309

[9] DELPHI Collaboration, DELSIM User's Guide, DELPHI 89-67 PROG 142.

[10] DELPHI Collaboration, DELSIM Reference Manual, DELPHI 89-68 PROG 143.

[11] T. Sjöstrand, High-energy-physics event generation with PYTHIA 5.7 and JETSET 7.4, Comp. Phys. Comm. 82(1994)74.

[12] DELPHI Collaboration, DELANA User's Guide, DELPHI 89-44 PROG 137.

[13] T. Sjöstrand, The Lund Monte Carlo for Jet Fragmentation and $e^{+} e^{-}$Jet Physics: JETSET Version 6.2, Comp. Phys. Comm. 39 (1986) 347.

[14] DELPHI collaboration, Performance of the DELPHI detector, Nuclear Instruments and Methods in Physics Research A378 (1996) 57.

[15] A. Sopczak, Status of Higgs Hunting at LEP - Five Years of Progress, CERNPPE/95-46.

[16] Searches Team B and Team C, Search for Higgs Bosons in Delphi at $\sqrt{s}=161 \mathrm{GeV}$, DELPHI 97-22 PHYS-678.

[17] Searches Team B and Team C, Update of the Neutral Higgs Boson Searches in Delphi at $\sqrt{s}=172 \mathrm{GeV}$, DELPHI 97-23 PHYS-679.

[18] DELPHI Collaboration, Search for neutral and charged Higgs bosons in $e^{+} e^{-}$collisions at $\sqrt{s}=161 \mathrm{GeV}$ and $172 \mathrm{GeV}$, To be submitted to Zeit. Phys. C.

[19] T. G. M. Malmgren, Iterative non-linear discriminant analysis - IDA 1.0, To be submitted to Comp. Phys. Comm. 